\title{
Student Epistemology About Mathematical Integration In A Physics Context: A Case Study
}

\author{
Joshua Von Korff ${ }^{1}$, Andrew Elby ${ }^{2}$, Dehui $\mathrm{Hu}^{3}$, N. Sanjay Rebello ${ }^{3}$ \\ ${ }^{1}$ Department of Physics, Georgia State University, 400 Science Annex, 29 Peachtree Ctr Ave N, Atlanta, GA 30303 \\ ${ }^{2}$ Department of Teaching \& Learning, Policy \& Leadership, University of Maryland, College Park, MD 20742 \\ ${ }^{3}$ Department of Physics, Kansas State University, 116 Cardwell Hall, Manhattan, KS 66506-2601
}

\begin{abstract}
During a sequence of interventions including a tutorial and a teaching interview, a student ("Bryce") showed evidence of understanding all the steps of Riemann-sum style reasoning about why displacement corresponds to the area under a velocity vs. time graph, which in turn corresponds to the integral of velocity over time. However, he does not view this reasoning as productive for explaining to someone why displacement corresponds to that area under the velocity vs. time curve. We argue that his view stems not primarily from conceptual or mathematical difficulties but from his epistemological stance toward learning integration. Although Bryce tries to mesh physical/conceptual reasoning and other pieces of mathematical formalism (such as simple equations), he views integration as something to be accepted and used. He therefore frames the Riemann sum reasoning as unneeded logical stepping stones rather than as a deeper explanation linking mathematical formalism to intuitive physical ideas.
\end{abstract}

Keywords: problem solving, mathematics, integration, epistemology

PACS: $01.40 . \mathrm{Fk}$

\section{INTRODUCTION}

The strategies that novices use to solve problems can stem from lack of conceptual understanding [1]. However, students' epistemologies can cause students not to access knowledge they have [2]. Thus students' solutions may reflect not only their conceptual understandings but also their epistemological stances - their views about what counts as knowing and understanding in a given context. We illustrate this point by analyzing an interview with Bryce, a student who had participated in a tutorial about integration in the context of kinematics. The tutorial was designed to facilitate student learning of what we call the 'layers' approach to physics problems requiring integration. After completing the tutorial however, we found that Bryce preferred a different approach to explaining why displacement is the area under a velocity vs. time graph. We argue that Bryce prefers the alternate approach not because he fails to understand the pieces of the layers argument, but because of his epistemological stance.

\section{METHODOLOGY}

This interview emerged from a larger study in which 52 introductory calculus-based physics students solved several problems in a tutorial about integration. The tutorial had three components. First, students solved a problem individually to find net displacement using a table of times, velocities, and accelerations. They might have done this by calculating $\Delta x=v \Delta t$ for each successive time step, then summing those small displacements. Second, students worked in groups of two to four on worksheets that guided them through the connection between the equation $W=F x$ and the fact that work is the area under the force vs. position curve. Finally, students again worked individually on the first problem. This time they could request a series of hints from a computer program.

Five students chose to participate in individual interviews a month later. The interviews were designed to find out what they remembered from the tutorial, and what they could reconstruct with and without help. As the interview progressed, students could review their own worksheets. The interviewer provided help only after giving the student a chance to perform a given task unassisted. Our interest in epistemology emerged from Bryce's responses and from the tutorials themselves, which highlighted arguments intended to persuade and explain.

Near the end of the interview, we asked Bryce the following question: Suppose someone only believes $\Delta x=v \Delta t$ but does not believe that position is the area under the velocity vs. time graph. Could you convince them of this? Before discussing Bryce's response, we lay out the framework we hoped Bryce would use to address this question. 


\section{THE 'LAYERS’ FRAMEWORK}

The layers framework, corresponding roughly to the notion of Riemann sums, is related to a framework for integration [3-5] and differentiation [6] by that name. It views the integral as a sum of products. For instance, the work integral could be decomposed as:

$W=d W_{1}+d W_{2}+\cdots=F_{1} d x+F_{2} d x+\ldots$

Equation (1) involves knowing about the sum layer and the product layer. Each layer corresponds to an equation. The product layer says that for a constant force, work can be found by multiplying force with displacement; $W=F x$. The product layer is also approximately true for very small displacements, $d W=F d x$, since the force is approximately constant over that displacement. The sum layer assumes that many small bits of work, over a trajectory, can be added to obtain the total work for the trajectory. Thus: $W=d W_{1}+d W_{2}+\cdots$. The sum and product layers together result in equation (1). This argument can be represented in graphical form, where $d W_{i}=F_{i} d x$ is the area of the $i^{\text {th }}$ narrow vertical rectangle, as in Fig. 1. Taking the limit as the rectangles become very narrow, we can demonstrate that the work is the area under the graph. Thus, work is the integral of force over displacement.

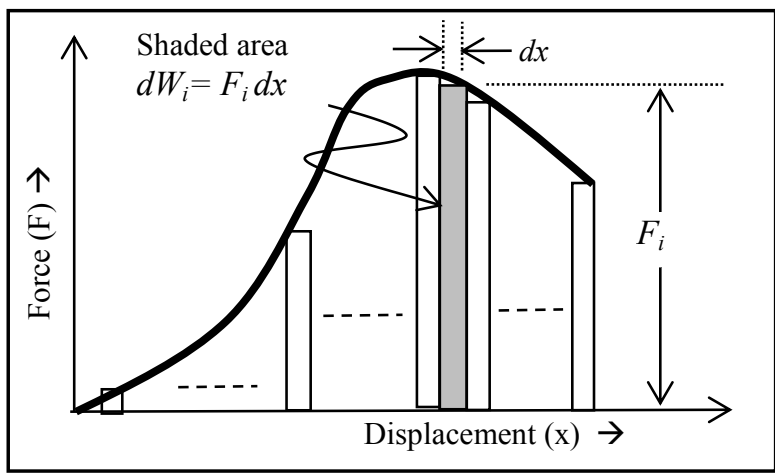

FIGURE 1. Graph representing the layers framework.

\section{BRYCE'S RESPONSE}

Given that Bryce completed our tutorial, we expected him to give something like the explanation above. Bryce had not only solved these worksheets a month earlier but also reconsidered them during the interview, when he and the interviewer discussed the implications of each page of the worksheet. However, the answer that Bryce proposed was different and is appealing due to its simplicity. We call it the "average height approach," since it involves the average height of a graph. To convince someone that displacement is the area under the velocity vs. time graph, the layers approach has the advantage that, starting with the equation $\Delta x=v \Delta t$, each step builds on the previous step to show why that displacement is the area under that graph, building on the intuitive idea that the distance covered during a tiny time interval is the given by the velocity multiplied by that time interval. By contrast, in the average height approach, the student simply jumps from the fact that displacement is the area under a constant (rectangular) velocity vs. time graph to the conclusion that displacement is the area under any velocity vs. time graph.

We were interested by the fact that Bryce showed evidence of understanding the steps of the layers argument, yet he unequivocally stated that he preferred the average height approach for convincing someone that displacement is the area under the velocity vs. time graph. We argue that Bryce prefers this approach not because he fails to understand the steps of the layers argument, but rather on epistemological grounds: he doesn't expect there to be an intuitive explanation for why integration is used to compute the displacement, and hence he views the steps of the layers argument as steps of a calculation or of a purely formal argument, not as steps of a physicallymeaningful explanation.

We now attempt to articulate Bryce's average height argument, first paraphrasing and then presenting Bryce's words. He talked about work before switching to displacement. The argument is that if the "average height" is multiplied by the width of the curve, we obtain the area under the graph. In essence, Bryce's argument contains three quantities which are purported to be equal: the area under the velocity vs. time graph $(A)$, the average height multiplied by the time $\left(h_{\text {avg }} \Delta t\right)$, and the displacement $\Delta \mathrm{x}$. (Although "height" in this case is velocity, we do not know if Bryce means "average velocity" in the usual sense.) Bryce is effectively saying that (a) $A=h_{\text {avg }} \Delta \mathrm{t}$ and (b) $h_{\text {avg }} \Delta \mathrm{t}=\Delta \mathrm{x}$. Combining (a) and (b) shows that $A=h_{\text {avg }} \Delta \mathrm{t}=\Delta \mathrm{x}$, so (c) $A=\Delta \mathrm{x}$. Applying this approach to a velocity vs. time graph, Bryce states that, "If you multiply this axis by this one [velocity and time], at defined heights, I mean, an average height, you're taking the area under the curve, which by the units and by the equation, you're getting your position."

Ironically, although Bryce uses the phrase "at defined heights," he never defines average height. Without this definition, his argument is incomplete. In fact, no definition of average height will complete Bryce's argument. If average height is defined as $A / \Delta \mathrm{t}$, then we can assume (a) but not (b), while if average height is defined as $\Delta \mathrm{x} / \Delta \mathrm{t}$, then we can assume (b) but not (a). Our point is that Bryce's 
explanation ends up having to assume the thing to be explained; it's an appeal to make a tempting generalization - that since area under the curve works for rectangles, it works for other shapes, too-rather than an explanation of why that generalization holds.

Earlier, talking about work, Bryce says, "you're finding area under the curve one way or another," pointing to " $W=F x$ " and to a graph of $F(x)$ with a horizontal line indicating "average height." Bryce is making the same appeal to generalizability as above, without a fuller explanation.

We have shown that Bryce's "average height" argument assumes its conclusion. This raises the question of whether Bryce prefers the average height approach because he doesn't understand the layers approach or for some other reason?

\section{BRYCE'S LAYERS UNDERSTANDING}

First, we would like to show that Bryce is capable of understanding integrals and areas in terms of the layers framework. Then, in the following section, we will ask what Bryce's actual motivation might be for selecting the average height approach.

The layers framework includes a product layer and a sum layer. Bryce showed evidence of understanding the product layer, stating that "work is the force times the distance," and later, "If you're multiplying force times distance, you're getting work no matter the situation." In the latter statement, Bryce is explaining why the area of a rectangle on a force vs. displacement graph is the work. He is also aware that a sum is involved in taking the area under the curve. For instance, he states that to find the displacement from the velocity graph, "you can just sum up the area under the curve and get your answer." Bryce views "summing up the area" as a useful procedure, and at one point he even draws a step-function graph of velocity versus time, stating that the area of one bar is the displacement, and the area of all bars is the total displacement.

In addition, after discussion with the interviewer and after reviewing some worksheets, Bryce articulates a layered explanation of the work integral. The interviewer points to a problem involving a spring which does $2 \mathrm{~J}$ of work on a mass, then $1 \mathrm{~J}$, then $1 \mathrm{~J}$ more as it extends. The problem asks how much work the spring does, and the answer can be found by summing the amounts of work. The interviewer asks, "What is this [problem] talking about?"

Bryce responds, "Finding like the work done over certain areas is just kind of multiplying, um, well actually you don't even have to really multiply um, cause it gives you the work done which is the force times the distance. So, if it had given you the distance travelled or the force done, then for each distance you could have found work that way and added them up." In this passage, Bryce describes a segmentation of a trajectory; by saying "for each distance," he implies a sequence of distances. Further, he recognizes that the work done during each segment can be found by multiplying "force times the distance," and these works are to be added up.

We are not claiming that Bryce's understanding of the layers approach is robust, simply that he understands the steps and in some circumstances can piece those steps together. One limitation of Bryce's understanding is that he tends to explain the layers approach one piece at a time. The passage quoted above, talking about the spring, is a rare moment in which he discusses sums and products together. During the interview, Bryce never uses the layers approach to explain why work is the integral of force over distance or the area under the force vs. distance graph. Although Bryce listens to the instructor offer such an explanation, using multiple representations, and shows strong evidence of following each step, Bryce himself never gives such an explanation. Our question therefore becomes, given that Bryce had the cognitive pieces in place to understand the layers explanation of integrals/areas and to piece together his own such explanation, why does he not do so?

\section{EPISTEMOLOGICAL EXPLANATION}

As described above, Bryce's preference for the average height approach cannot be fully explained by a lack of knowledge about layers. We now argue that his epistemology plays a role. We begin by pointing out that the layers argument, to experts, is a derivation with explanatory power. Bryce shows evidence of doubting the value of derivations. When the interviewer asks whether Bryce remembers the proof that the integral of velocity over time is displacement, Bryce states that he "didn't really pay attention" when his high school teacher explained it. He didn't recall the proof, but says "I'm pretty sure it took [the high school teacher] a while" to explain the proof, indicating he perceived the proof to be long.

Bryce portrayed himself as not valuing proofs and derivations in a second instance as well. After he considered the two approaches for explaining the velocity integral, Bryce stated a preference for the average height approach, because "I tend to believe things that are, kind of told to me, like in an equation or whatever. So I would personally take the jump to ... from one to the average height. But for a lot of people that like wanna, like know like where you're coming from when you say this? [The layers approach] would be a much better way to explain it." Bryce notes that 
people other than himself might value the longer chain of reasoning because it's "like a more logical stepping stone from one place to the next [...] it's I guess easier to like walk through it, like step by step. And make it [...] make sense that way." Thus, Bryce is capable of seeing things from the layers point of view and even defending it on epistemological grounds ("a more logical stepping stone"), but ultimately he would "personally" choose the average height approach. Notice also that Bryce's defense of the layers approach mentions its logical step-by-step nature but not the way it makes conceptual connections between sums, products, graphs, and kinematic quantities.

Bryce's stance is partly epistemological, as he weighs the value of believing what one is told, logical stepping stones, and making sense. He seems to be saying that he wants to just "believe" formulas rather than doing extra work to understand where they came from. Elsewhere in the interview, when asked how he would explain the integral to another student, Bryce sometimes states that he would just tell them without explanation: "You could just kind of straight up tell 'em that the integral of velocity is position, and so on and so forth with acceleration as well. And if they don't believe that, then they're just stubborn."

This epistemological stance does not characterize Bryce's view towards mathematical formalism more generally, however. He frequently constructs explanations to help him understand formulas. For example, when the interviewer asks Bryce how he knows that pressure equals force over area and not force times area, Bryce says: "I just kind of step back and think about it," and makes an attempt at explaining. By contrast, when the interviewer asks Bryce, early in the interview, how he knows that displacement is the integral of velocity, Bryce says it's "just something I learned in high school I guess."

This seeming inconsistency suggests that Bryce holds context-dependent epistemological stances rather than context-free beliefs [7]. We lack sufficient data to characterize the cues that push Bryce towards accepting knowledge from authority versus trying to make sense of it ("step back and think about it"). Inconclusive evidence-which a later interview could support or refute-suggests that derivations tend to push Bryce away from conceptual/intuitive sensemaking. For instance, he can see how the steps of the layers argument might hold explanatory power for other people, but not so much for him personally. The formal complexity of integration (as opposed to, say, the equation $p=F / A$ ) may also play a role.

Subtleties aside, however, our main argument is that, in the context of thinking about integration and the layers argument, Bryce tends to view the formalism as something to accept rather than to make conceptual/intuitive sense of, and hence he treats the layers argument as "logical stepping stone[s]" but not as an explanation linking intuitive physics ideas to mathematical formalism. This, we argue, helps to explain his preference for the average height explanation of why displacement is the area under a velocity vs. time graph, even though that "explanation" (from our perspective) assumes what it is trying to explain.

\section{LIMITATIONS \& CONCLUSIONS}

Although our case study cannot establish whether Bryce is "typical," we have shown how one student's trouble with understanding the physical meaning of integration stemmed not just from previously documented conceptual and mathematical difficulties but also from the epistemological stance he took toward learning integration. Although Bryce connects mathematical formalism to physics meaning in other contexts, the topic of integration seems to bring out a more "knowledge from authority" stance [8].

\section{ACKNOWLEDGMENTS}

This work is supported in part by National Science Foundation grant 0816207. Opinions, expressed are of the authors and not necessarily of the Foundation.

\section{REFERENCES}

1. L. Hsu, E. Brewe, T. M. Foster and K. A. Harper, American Journal of Physics 72 (9), 1147-1156 (2004).

2. A. Elby, Physics Education Research: A Supplement to the American Journal of Physics 69 (7), S54-S64 (2001).

3. P. W. Thompson and J. Silverman, in Making the connection: Research and teaching in undergraduate mathematics, edited by M. P. Carlson and C. Rasmussen (Washington, DC, 2008), Vol. 43-52.

4. V. Sealey, in Proceedings of the Twenty Eighth Annual Meeting of the North American Chapter of the International Group for the Psychology of Mathematics Education 2, edited by S. Alatorre, J. L. Cortina, M. Sáiz and A. Méndez (Mérida, Mexico: Universidad Pedagógica Nacional, 2006), pp. 46-53

5. J. Von Korff and N. S. Rebello, Physical Review Special Topics - Physics Education Research 8 (1), 010125 (2011).

6. M. Zandieh, in Research in Collegiate Mathematics Education IV, edited by E. Dubinsky, A. H. Schoenfeld and J. Kaput (American Mathematical Society, Providence, RI, 2000), pp. 103

7. D. Hammer, American Journal of Physics - Physics Education Research Supplement 68 (7), S52-S59 (2000).

8. D. Hammer, Cognition and Instruction 12 (2), 151-183 (1994). 